\title{
CAUCHY APPROXIMATION FOR SUMS OF INDEPENDENT RANDOM VARIABLES
}

\author{
K. NEAMMANEE
}

Received 6 August 2002

We use Stein's method to find a bound for Cauchy approximation. The random variables which are considered need to be independent.

2000 Mathematics Subject Classification: 60F05, 60G50.

1. Introduction. In Stein's work [19], the aim was to show convergence in distribution to the normal. His technique was novel. Stein's technique was free from Fourier methods and relied instead on the elementary differential equation

$$
f^{\prime}(w)-w f(w)=h(x)-N h \quad(w \in \mathbb{R}),
$$

where $h: \mathbb{R} \rightarrow \mathbb{R}$ is such that

$$
\int_{-\infty}^{\infty}|h(x)| e^{-(1 / 2) x^{2}} d x<\infty
$$

and $N h=E(h(Z))$, where $Z \sim N(0,1)$.

Stein's method was extended from normal distribution to the Poisson distribution by Chen [9]. Stein's equation for Poisson with parameter $\lambda$ is

$$
\lambda f(w+1)-w f(w)=h(w)-P_{\lambda} h \quad\left(w \in \mathbb{Z}^{+}\right),
$$

where $P_{\lambda} h=E(h(Z)), Z \sim \operatorname{Poi}(\lambda)$.

Since then, Stein's method has found considerable applications in combinatorics, probability, and statistics. Recent literature pertaining to this method includes Arratia et al. [1, 2], Baldi and Rinott [3], Barbour [4, 5], Barbour et al. [6], Bolthausen and Götze [7], Chen [10, 11], Goldstein and Reinert [12], Goldstein and Rinott [13], Götze [14], and Green [15]; the work of Holst and Janson [16] gives an excellent account of this method. In this paper, we further develop the Stein technique to bound errors for a Cauchy approximation to the distribution of $W$, the sum of independent random variables. In fact, there are some literatures (e.g., Boonyasombut and Shapiro [8], Neammanee [17], and Shapiro [18]) give a bound of Cauchy approximation in some kind of random variables. But they used Fourier methods. 
This paper is organized as follows. Main results are stated in Section 2. Proof of main results is in Section 3, while an example is given in Section 4 .

2. Main results. At the heart of Stein's method lies a Stein equation. For example,

$$
\begin{array}{rlrl}
f^{\prime}(w)-w f(w) & =g(w), & w & \in \mathbb{R}, \\
\lambda f(w+1)-w f(w) & =g(w), & w \in \mathbb{Z}^{+}
\end{array}
$$

are Stein equations for normal and Poisson distribution, respectively.

Let $\mathscr{H}=\left\{h: \mathbb{R} \rightarrow \mathbb{R} \mid \int_{-\infty}^{\infty}\left(|h(x)| /\left(1+x^{2}\right)\right) d x<\infty\right\}$, and for each $h \in \mathcal{H}$,

$$
\operatorname{Cau}(h)=\frac{1}{\pi} \int_{-\infty}^{\infty} \frac{h(x)}{1+x^{2}} d x .
$$

The Stein equation for Cauchy distribution $F$

$$
F(x)=\frac{1}{\pi} \int_{-\infty}^{x} \frac{1}{1+t^{2}} d t
$$

is

$$
f^{\prime}(w)-\frac{2 w f(w)}{1+w^{2}}=h(w)-\operatorname{Cau}(h)
$$

It is easy to check that a solution of (2.4) is $U_{h}: \mathbb{R} \rightarrow \mathbb{R}$ defined by

$$
U_{h}(w)=\left(1+w^{2}\right) \int_{-\infty}^{w} \frac{h(x)-\operatorname{Cau}(h)}{1+x^{2}} d x .
$$

Fix $w_{0} \in \mathbb{R}$, and choose $h$ to be the indicator function $I_{\left(-\infty, w_{0}\right]}$ which is defined by

$$
I_{\left(-\infty, w_{0}\right]}(w)= \begin{cases}1 & \text { if } w \leq w_{0} \\ 0 & \text { if } w>w_{0}\end{cases}
$$

Let $f_{w_{0}}=U_{I_{\left(-\infty, w_{0}\right.}}$. Then, by (2.2), (2.3), and (2.5), we see that

$$
f_{w_{0}}(w)= \begin{cases}\pi\left(1+w^{2}\right) F(w)\left(1-F\left(w_{0}\right)\right) & \text { if } w \leq w_{0} \\ \pi\left(1+w^{2}\right) F\left(w_{0}\right)(1-F(w)) & \text { if } w \geq w_{0}\end{cases}
$$

The broad idea of Stein's argument is as follows. First, for any $w_{0} \in \mathbb{R}$, a function $f_{w_{0}}: \mathbb{R} \rightarrow \mathbb{R}$ is constructed to solve (2.4) when $h$ is the indicator function $I_{\left(-\infty, w_{0}\right]}$. Replacing $w$ by $W$, for any random variable $W$, it therefore follows that the difference between $P\left(W \leq w_{0}\right)$ and $F\left(w_{0}\right)$ can be expressed as

$$
E\left\{f_{w_{0}}^{\prime}(W)-\frac{2 W f_{w_{0}}(W)}{1+W^{2}}\right\}
$$

The main results are the following. 
THEOREM 2.1. Let $X_{1}, X_{2}, \ldots, X_{n}$ be independent random variables with $E X_{i}$ $=0, E X_{i}^{2}=\sigma_{i}^{2}$, and $E\left|X_{i}\right|^{4}<\infty$. Then,

$$
\begin{aligned}
\mid P(W & \left.\leq w_{0}\right)-F\left(w_{0}\right) \mid \\
\leq & 3 \sqrt{E\left[1-\sum_{i=1}^{n} \frac{\sigma_{i}^{2}+X_{i}^{2}}{1+W^{2}}\right]^{2}} \\
& +4 \pi \min \left\{\sum_{i=1}^{n} \sigma_{i}^{2}, 2 \sqrt{n \sum_{i=1}^{n} \sigma_{i}^{2} \sum_{i=1}^{n} E\left|X_{i}\right|^{4}}\right\} F\left(w_{0}\right)\left(1-F\left(w_{0}\right)\right) \\
& +C \sum_{i=1}^{n} E\left|X_{i}\right|^{3},
\end{aligned}
$$

when $W=X_{1}+X_{2}+\cdots+X_{n}$.

COROLLARY 2.2. Let $Y_{1}, Y_{2}, \ldots, Y_{n}$ be identically independent random variables with zero means $E Y_{i}^{2}=1 / 2$ and $E\left|Y_{i}\right|^{5}<\infty$. Let $X_{i}=Y_{i} / \sqrt{n}$ and $W=$ $X_{1}+X_{2}+\cdots+X_{n}$. Then,

$$
\left|P\left(W \leq w_{0}\right)-F\left(w_{0}\right)\right|<\frac{C}{\sqrt[4]{n}}+C \min \left\{\frac{1}{2}, \sqrt{2} \sqrt{E Y_{i}^{4}}\right\} F\left(w_{0}\right)\left(1-F\left(w_{0}\right)\right) .
$$

Throughout this paper, $C$ stands for an absolute constant with possibly different values in different places.

3. Proof of main results. Before we prove the main results, we need the following lemmas.

LEMMA 3.1. For any real numbers $w_{0}$ and $w$,

(1) $\left|f_{w_{0}}(w) /\left(1+w^{2}\right)\right| \leq \pi F\left(w_{0}\right)\left(1-F\left(w_{0}\right)\right)$

(2) $\left|f_{w_{0}}^{\prime}(w)\right| \leq 3$

(3) $\left|f_{w_{0}}^{\prime \prime}(w)\right| \leq 3+2 \pi$

(4) $\left|\left(f_{w_{0}}^{\prime}(w) /\left(1+w^{2}\right)\right)^{\prime}\right| \leq 6+2 \pi$

(5) $\left|\left(w f_{w_{0}}(w) /\left(1+w^{2}\right)^{2}\right)^{\prime}\right| \leq 3+5 \pi$.

Proof. (1) follows directly from (2.7).

(2) Before we start the proof, we need the following inequalities:

$$
\begin{gathered}
-\frac{1}{\pi} \leq w F(w) \leq 0 \quad \text { for } w \leq 0, \\
0 \leq w(1-F(w)) \leq \frac{1}{\pi} \quad \text { for } w>0
\end{gathered}
$$


To show (3.1), we define $g$ on $(-\infty, 0]$ by $g(w)=w F(w)$. Since $g^{\prime \prime}(w)=$ $2 / \pi\left(1+w^{2}\right)^{2}>0, g^{\prime}$ is increasing. From this fact and the fact that

$$
\lim _{w \rightarrow-\infty} g^{\prime}(w)=\lim _{w \rightarrow-\infty} \frac{1}{\pi}\left(\frac{w}{1+w^{2}}+\arctan w+\frac{\pi}{2}\right)=0,
$$

we have $g^{\prime} \geq 0$. Hence, $g$ is increasing and

$$
-\frac{1}{\pi}=\lim _{t \rightarrow-\infty} g(t) \leq g(w) \leq g(0)=0
$$

for any $w \leq 0$. So (3.1) holds. To show (3.2), we can apply the same argument to the function $\tilde{g}$ on $[0, \infty)$ which is defined by $\tilde{g}(w)=w(1-C(w))$. Since $f_{w_{0}}(w)=f_{-w_{0}}(-w)$, it suffices to prove the lemma in the case where $w_{0} \geq 0$. By (2.7), we have

$$
\begin{aligned}
\left|f_{w_{0}}^{\prime}(w)\right| & = \begin{cases}\left|\left(1-F\left(w_{0}\right)\right)(1+2 \pi w F(w))\right| & \text { if } w \leq 0, \\
\left|F\left(w_{0}\right)(-1+2 \pi w(1-F(w)))\right| & \text { if } w \geq w_{0}\end{cases} \\
& \leq \begin{cases}1+2 \pi|w F(w)| & \text { if } w \leq 0, \\
1+2 \pi|w(1-F(w))| & \text { if } w \geq w_{0}\end{cases} \\
& \leq \begin{cases}3 & \text { if } w \leq 0, \\
3 & \text { if } w \geq w_{0},\end{cases}
\end{aligned}
$$

where we have used the fact that $0 \leq F(w) \leq 1$ in the first inequality and (3.1) and (3.2) in the second inequality. In the case where $0 \leq w \leq w_{0}$, by monotonicity of $F$ and (3.2), we see that

$$
\begin{aligned}
0 & \leq f_{w_{0}}^{\prime}(w) \\
& =\left(1-F\left(w_{0}\right)\right)+2 \pi\left(1-F\left(w_{0}\right)\right) w F(w) \\
& \leq 1+2 \pi(1-F(w)) w \leq 3
\end{aligned}
$$

Hence, (2) follows from (3.5) and (3.6).

(3) follows immediately from (2) and the fact that

$$
f_{w_{0}}^{\prime \prime}(w)=\frac{2 w}{1+w^{2}} f_{w_{0}}^{\prime}(w)+\frac{2\left(1-w^{2}\right)}{\left(1+w^{2}\right)^{2}} f_{w_{0}}(w) .
$$

(4) and (5) follow from (2) and (3) and the facts that

$$
\begin{aligned}
\left(\frac{f_{w_{0}}^{\prime}(w)}{1+w^{2}}\right)^{\prime} & =\frac{f_{w_{0}}^{\prime \prime}(w)}{1+w^{2}}-\frac{2 w f_{w_{0}}^{\prime}(w)}{\left(1+w^{2}\right)^{2}} \\
\left(\frac{w f_{w_{0}}(w)}{\left(1+w^{2}\right)^{2}}\right)^{\prime} & =\frac{w f_{w_{0}}^{\prime}(w)+f_{w_{0}}(w)}{\left(1+w^{2}\right)^{2}}-\frac{4 w^{2} f_{w_{0}}(w)}{\left(1+w^{2}\right)^{3}}
\end{aligned}
$$


LEMMA 3.2. Let $(W, \widetilde{W})$ be an exchangeable pair of random variables, that is,

$$
P(W \in B, \widetilde{W} \in \widetilde{B})=P(W \in \widetilde{B}, \widetilde{W} \in B)
$$

for any Borel sets $B$ and $\widetilde{B}$ on $\mathbb{R}$, and there exists $\lambda>0$ such that

$$
E^{W} \widetilde{W}=(1-\lambda) W, \quad E|\widetilde{W}-W|^{2}<\infty,
$$

where $E^{W} \widetilde{W}$ is the conditional expectation of $\widetilde{W}$ with respect to $W$. Then,

$$
E\left[\frac{2 W f(W)}{1+W^{2}}-\frac{1}{\lambda}(\widetilde{W}-W)\left(\frac{f(\widetilde{W})}{1+\widetilde{W}^{2}}-\frac{f(W)}{1+W^{2}}\right)\right]=0
$$

for any function $f: \mathbb{R} \rightarrow \mathbb{R}$, for which there exists $C>0$ such that for all $w \in \mathbb{R}$,

$$
|f(w)| \leq C\left(1+w^{2}\right) .
$$

Moreover,

$$
P\left(W \leq w_{0}\right)=C\left(w_{0}\right)+E\left[f_{w_{0}}^{\prime}(W)-\frac{1}{\lambda}(\widetilde{W}-W)\left(\frac{f_{w_{0}}(\widetilde{W})}{1+\widetilde{W}^{2}}-\frac{f_{w_{0}}(W)}{1+W^{2}}\right)\right]
$$

for any $w_{0} \in \mathbb{R}$.

Proof. Define $F: \mathbb{R}^{2} \rightarrow \mathbb{R}$ by

$$
F(w, \widetilde{w})=(\widetilde{w}-w)\left[\frac{f(\widetilde{w})}{1+\widetilde{w}^{2}}+\frac{f(w)}{1+w^{2}}\right]
$$

Then, $F$ is antisymmetric, that is, $F(w, \widetilde{w})=-F(\widetilde{w}, w)$. By Stein [20, pages 9-10], we have $E F(W, \widetilde{W})=0$, which implies that

$$
\begin{aligned}
0 & =E(\widetilde{W}-W)\left[\frac{f(\widetilde{W})}{1+\widetilde{W}^{2}}+\frac{f(W)}{1+W^{2}}\right] \\
& =E(\widetilde{W}-W)\left\{\frac{2 f(W)}{1+W^{2}}+\left[\frac{f(\widetilde{W})}{1+\widetilde{W}^{2}}-\frac{f(W)}{1+W^{2}}\right]\right\} \\
& =2 E\left(E^{W} \widetilde{W}-W\right) \frac{f(W)}{1+W^{2}}+E(\widetilde{W}-W)\left[\frac{f(\widetilde{W})}{1+\widetilde{W}^{2}}-\frac{f(W)}{1+W^{2}}\right] \\
& =-\lambda E\left(\frac{2 W f(W)}{1+W^{2}}\right)+E(\widetilde{W}-W)\left[\frac{f(\widetilde{W})}{1+\widetilde{W}^{2}}-\frac{f(W)}{1+W^{2}}\right] \\
& =E\left[\frac{2 W f(W)}{1+W^{2}}-\frac{1}{\lambda}(\widetilde{W}-W)\left[\frac{f(\widetilde{W})}{1+\widetilde{W^{2}}}-\frac{f(W)}{1+W^{2}}\right]\right] .
\end{aligned}
$$

Then, (3.11) holds and (3.13) follows from (3.11) and (2.4) when $h=I_{\left(-\infty, w_{0}\right]}$. 
LEMmA 3.3. Let $(W, \widetilde{W})$ be an exchangeable pair of random variables such that

$$
E^{W} \widetilde{W}=(1-\lambda) W, \quad E|\widetilde{W}-W|^{2}<\infty
$$

with $\lambda>0$. Then, for any $w_{0} \in \mathbb{R}$,

$$
\begin{aligned}
P(W \leq & \left.w_{0}\right) \\
= & C\left(w_{0}\right)+E f_{w_{0}}^{\prime}(W)\left[1-\frac{1}{\lambda} E^{W} \frac{(\widetilde{W}-W)^{2}}{1+W^{2}}\right]+\frac{2}{\lambda} \frac{E(\widetilde{W}-W)^{2} W f_{w_{0}}(W)}{\left(1+W^{2}\right)^{2}} \\
+ & \frac{1}{\lambda} \int_{-\infty}^{\infty} E(\widetilde{W}-W)\left(w-\frac{W+\widetilde{W}}{2}\right) \\
& \times[I(w \leq \widetilde{W})-I(w \leq W)]\left(\frac{f_{w_{0}}^{\prime}(w)}{1+w^{2}}\right)^{\prime} d w \\
- & \frac{2}{\lambda} \int_{-\infty}^{\infty} E(\widetilde{W}-W)\left(w-\frac{W+\widetilde{W}}{2}\right) \\
& \times[I(w \leq \widetilde{W})-I(w \leq W)]\left(\frac{w f_{w_{0}}(w)}{\left(1+w^{2}\right)^{2}}\right)^{\prime} d w .
\end{aligned}
$$

Proof. Let $w_{0} \in \mathbb{R}$. For $W<\widetilde{W}$, we see that

$$
\begin{aligned}
\frac{f_{w_{0}}(\widetilde{W})}{1+\widetilde{W}^{2}}-\frac{f_{w_{0}}(W)}{1+W^{2}}-\frac{(\widetilde{W}-W) f_{w_{0}}^{\prime}(W)}{1+W^{2}}+\frac{2(\widetilde{W}-W) W f_{w_{0}}(W)}{\left(1+W^{2}\right)^{2}} \\
=\int_{W}^{\widetilde{W}}\left[\left(\frac{f_{w_{0}}(w)}{1+w^{2}}\right)^{\prime}-\frac{f_{w_{0}}^{\prime}(W)}{1+W^{2}}+\frac{2 W f_{w_{0}}(W)}{\left(1+W^{2}\right)^{2}}\right] d w \\
=\int_{W}^{\widetilde{W}}\left[\frac{f_{w_{0}}^{\prime}(w)}{1+w^{2}}-\frac{2 w f_{w_{0}}(w)}{\left(1+w^{2}\right)^{2}}-\frac{f_{w_{0}}^{\prime}(W)}{1+W^{2}}+\frac{2 W f_{w_{0}}(W)}{\left(1+W^{2}\right)^{2}}\right] d w \\
=\int_{W}^{\widetilde{W}} \int_{W}^{w}\left(\frac{f_{w_{0}}^{\prime}(y)}{1+y^{2}}\right)^{\prime} d y d w-2 \int_{W}^{\widetilde{W}} \int_{W}^{w}\left(\frac{y f_{w_{0}}(y)}{\left(1+y^{2}\right)^{2}}\right)^{\prime} d y d w \\
=\int_{W}^{\widetilde{W}} \int_{y}^{\widetilde{W}}\left(\frac{f_{w_{0}}^{\prime}(y)}{1+y^{2}}\right)^{\prime} d w d y-2 \int_{W}^{\widetilde{W}} \int_{y}^{\widetilde{W}}\left(\frac{y f_{w_{0}}(y)}{\left(1+y^{2}\right)^{2}}\right)^{\prime} d w d y \\
=\int_{W}^{\widetilde{W}}(\widetilde{W}-y)\left(\frac{f_{w_{0}}^{\prime}(y)}{1+y^{2}}\right)^{\prime} d y-2 \int_{W}^{\widetilde{W}}(\widetilde{W}-y)\left(\frac{y f_{w_{0}}(y)}{\left(1+y^{2}\right)^{2}}\right)^{\prime} d y,
\end{aligned}
$$

and by the same argument we can show that

$$
\begin{aligned}
& \frac{f_{w_{0}}(\widetilde{W})}{1+\widetilde{W}^{2}}-\frac{f_{w_{0}}(W)}{1+W^{2}}-\frac{(\widetilde{W}-W) f_{w_{0}}^{\prime}(W)}{1+W^{2}}+\frac{2(\widetilde{W}-W) W f_{w_{0}}(W)}{\left(1+W^{2}\right)^{2}} \\
& =\int_{\widetilde{W}}^{W}(w-\widetilde{W})\left(\frac{f_{w_{0}}^{\prime}(w)}{1+w^{2}}\right)^{\prime} d w-2 \int_{\widetilde{W}}^{W}(w-\widetilde{W})\left(\frac{w f_{w_{0}}(w)}{\left(1+w^{2}\right)^{2}}\right)^{\prime} d w
\end{aligned}
$$

for $\widetilde{W}<W$. 
So,

$$
\begin{gathered}
\frac{f_{w_{0}}(\widetilde{W})}{1+\widetilde{W}^{2}}-\frac{f_{w_{0}}(W)}{1+W^{2}}-\frac{(\widetilde{W}-W) f_{w_{0}}^{\prime}(W)}{1+W^{2}}+\frac{2(\widetilde{W}-W) W f_{w_{0}}(W)}{\left(1+W^{2}\right)^{2}} \\
=\int_{-\infty}^{\infty}(\widetilde{W}-w)[I(w \leq \widetilde{W})-I(w \leq W)]\left(\frac{f_{w_{0}}^{\prime}(w)}{1+w^{2}}\right)^{\prime} d w \\
\quad-2 \int_{-\infty}^{\infty}(\widetilde{W}-w)[I(w \leq \widetilde{W})-I(w \leq W)]\left(\frac{w f_{w_{0}}(w)}{\left(1+w^{2}\right)^{2}}\right)^{\prime} d w .
\end{gathered}
$$

By Lemma 3.2, we have

$$
\begin{aligned}
& P\left(W \leq w_{0}\right) \\
& =C\left(w_{0}\right)+E\left[f_{w_{0}}^{\prime}(W)-\frac{1}{\lambda} \frac{f_{w_{0}}^{\prime}(W)(\widetilde{W}-W)^{2}}{1+W^{2}}+\frac{1}{\lambda} \frac{f_{w_{0}}^{\prime}(W)(\widetilde{W}-W)^{2}}{1+W^{2}}\right. \\
& +\frac{2}{\lambda} \frac{(\widetilde{W}-W)^{2} W f_{w_{0}}(W)}{\left(1+W^{2}\right)^{2}}-\frac{2}{\lambda} \frac{(\widetilde{W}-W)^{2} W f_{w_{0}}(W)}{\left(1+W^{2}\right)^{2}} \\
& \left.-\frac{1}{\lambda}(\widetilde{W}-W)\left[\frac{f_{w_{0}}(\widetilde{W})}{1+\widetilde{W}^{2}}-\frac{f_{w_{0}}(W)}{1+W^{2}}\right]\right] \\
& =C\left(w_{0}\right)+E f_{w_{0}}^{\prime}(W)-\frac{1}{\lambda} E E^{W} \frac{f_{w_{0}}^{\prime}(W)(\widetilde{W}-W)^{2}}{1+W^{2}} \\
& +\frac{2}{\lambda} \frac{E(\widetilde{W}-W)^{2} W f_{w_{0}}(W)}{\left(1+W^{2}\right)^{2}}-\frac{1}{\lambda} E(\widetilde{W}-W) \\
& \times\left[\frac{f_{w_{0}}(\widetilde{W})}{1+\widetilde{W}^{2}}-\frac{f_{w_{0}}(W)}{1+W^{2}}-\frac{(\widetilde{W}-W) f_{w_{0}}^{\prime}(W)}{1+W^{2}}+\frac{2(\widetilde{W}-W) W f_{w_{0}}(W)}{\left(1+W^{2}\right)^{2}}\right] \\
& =C\left(w_{0}\right)+E\left[f_{w_{0}}^{\prime}(W)\left\{1-\frac{1}{\lambda} E^{W} \frac{(\widetilde{W}-W)^{2}}{1+W^{2}}\right\}\right] \\
& +\frac{2}{\lambda} \frac{E(\widetilde{W}-W)^{2} W f_{w_{0}}(W)}{\left(1+W^{2}\right)^{2}}-\frac{1}{\lambda} E(\widetilde{W}-W) \\
& \times\left[\frac{f_{w_{0}}(\widetilde{W})}{1+\widetilde{W}^{2}}-\frac{f_{w_{0}}(W)}{1+W^{2}}-\frac{(\widetilde{W}-W) f_{w_{0}}^{\prime}(W)}{1+W^{2}}+\frac{2(\widetilde{W}-W) W f_{w_{0}}(W)}{\left(1+W^{2}\right)^{2}}\right] \\
& =C\left(w_{0}\right)+E\left[f_{w_{0}}^{\prime}(W)\left\{1-\frac{1}{\lambda} E^{W} \frac{(\widetilde{W}-W)^{2}}{1+W^{2}}\right\}\right]+\frac{2}{\lambda} \frac{E(\widetilde{W}-W)^{2} W f_{w_{0}}(W)}{\left(1+W^{2}\right)^{2}} \\
& -\frac{1}{\lambda} E(\widetilde{W}-W) \int_{-\infty}^{\infty}(\widetilde{W}-w)[I(w \leq \widetilde{W})-I(w \leq W)]\left(\frac{f_{w_{0}}^{\prime}(w)}{1+w^{2}}\right)^{\prime} d w \\
& +\frac{2}{\lambda} E(\widetilde{W}-W) \int_{-\infty}^{\infty}(\widetilde{W}-w)[I(w \leq \widetilde{W})-I(w \leq W)]\left(\frac{w f_{w_{0}}(w)}{\left(1+w^{2}\right)^{2}}\right)^{\prime} d w,
\end{aligned}
$$

where we have used (3.20) in the last equality. 
For fixed $w$, we define $F: \mathbb{R}^{2} \rightarrow \mathbb{R}$ by

$$
F(x, \tilde{x})=(x-\tilde{x})\left(\frac{x-\tilde{x}}{2}\right)[I(w \leq \tilde{x})-I(w \leq x)] .
$$

Then, $F$ is antisymmetric. Since $W$ and $\widetilde{W}$ are exchangeable, $E F(W, \widetilde{W})=0$. Thus,

$$
\begin{aligned}
E(\widetilde{W}-W)(w-\widetilde{W})[I(w \leq \widetilde{W})-I(w \leq W)] \\
=E(\widetilde{W}-W)\left(w-\frac{W+\widetilde{W}}{2}+\frac{W-\widetilde{W}}{2}\right)[I(w \leq \widetilde{W})-I(w \leq W)] \\
=E(\widetilde{W}-W)\left(w-\frac{W+\widetilde{W}}{2}\right)[I(w \leq \widetilde{W})-I(w \leq W)]-E F(W, \widetilde{W}) \\
=E(\widetilde{W}-W)\left(w-\frac{W+\widetilde{W}}{2}\right)[I(w \leq \widetilde{W})-I(w \leq W)] .
\end{aligned}
$$

By (3.21) and (3.23), the lemma is proved.

Proof of Theorem 2.1. Let $X_{1}, X_{2}, \ldots, X_{n}$ be independent random variables and $W=X_{1}+X_{2}+\cdots+X_{n}$. In order to prove the theorem, we introduce additional random variables $I, \widetilde{X}_{1}, \tilde{X}_{2}, \ldots, \widetilde{X}_{n}$, and $\widetilde{W}$ defined in the following way. The random variables $I, X_{1}, X_{2}, \ldots, X_{n}, \tilde{X}_{1}, \tilde{X}_{2}, \ldots, \tilde{X}_{n}$ are independent, $I$ is uniformly distributed over the index set $\{1,2, \ldots, n\}$, each $\tilde{X}_{i}$ has the same distribution as the corresponding $X_{i}$ and $\widetilde{W}=W+\left(\widetilde{X}_{I}-X_{I}\right)$. Then, $(W, \widetilde{W})$ is an exchangeable pair. We note that

$$
\begin{aligned}
E^{W} \widetilde{W} & =W+E^{W} \tilde{X}_{I}-E^{W} X_{I}=W-\frac{1}{n} \sum_{i=1}^{n} X_{i}=\left(1-\frac{1}{n}\right) W \\
E|\widetilde{W}-W|^{2} & =E\left|\tilde{X}_{I}-X_{I}\right|^{2}=\frac{1}{n} \sum_{i=1}^{n} E\left|\tilde{X}_{i}-X_{i}\right|^{2}=\frac{2}{n} \sum_{i=1}^{n} \sigma_{i}^{2} .
\end{aligned}
$$

Then, the assumptions of Lemma 3.3 are satisfied with $\lambda=1 / n$. Moreover, we know that

$$
\begin{aligned}
& E|\widetilde{W}-W|^{3}=E\left|\tilde{X}_{I}-X_{I}\right|^{3}=\frac{1}{n} \sum_{i=1}^{n} E\left|\tilde{X}_{i}-X_{i}\right|^{3} \leq \frac{8}{n} \sum_{i=1}^{n} E\left|X_{i}\right|^{3}, \\
& E|\widetilde{W}-W|^{4}=E\left|\tilde{X}_{I}-X_{I}\right|^{4}=\frac{1}{n} \sum_{i=1}^{n} E\left|\tilde{X}_{i}-X_{i}\right|^{4} \leq \frac{16}{n} \sum_{i=1}^{n} E\left|X_{i}\right|^{4} .
\end{aligned}
$$


To prove the theorem, let $w_{0} \in \mathbb{R}$. By Lemma 3.3, we obtain

$$
\begin{aligned}
& \left|P\left(W \leq w_{0}\right)-C\left(w_{0}\right)\right| \\
& \leq \sup _{w \in \mathbb{R}}\left|f_{w_{0}}^{\prime}(w)\right| E\left|1-n E^{W} \frac{(\widetilde{W}-W)^{2}}{1+W^{2}}\right|+2 n\left|\frac{E(\widetilde{W}-W)^{2} W f_{w_{0}}(W)}{\left(1+W^{2}\right)^{2}}\right| \\
& +n \sup _{w \in \mathbb{R}}\left|\left(\frac{f_{w_{0}}^{\prime}(w)}{1+w^{2}}\right)^{\prime}\right| E \int_{-\infty}^{\infty}|\widetilde{W}-W|\left|\left(w-\frac{W+\widetilde{W}}{2}\right)\right| \\
& \times|[I(w \leq \widetilde{W})-I(w \leq W)]| d w \\
& +2 n \sup _{w \in \mathbb{R}}\left|\left(\frac{w f_{w_{0}}(w)}{\left(1+w^{2}\right)^{2}}\right)^{\prime}\right| E \int_{-\infty}^{\infty}|\widetilde{W}-W|\left|\left(w-\frac{W+\widetilde{W}}{2}\right)\right| \\
& \times|[I(w \leq \widetilde{W})-I(w \leq W)]| d w \\
& \leq \sup _{w \in \mathbb{R}}\left|f_{w_{0}}^{\prime}(w)\right| E\left|1-n E^{W} \frac{(\widetilde{W}-W)^{2}}{1+W^{2}}\right|+2 n\left|\frac{E(\widetilde{W}-W)^{2} W f_{w_{0}}(W)}{\left(1+W^{2}\right)^{2}}\right| \\
& +\left(n \sup _{w \in \mathbb{R}}\left|\left(\frac{f_{w_{0}}^{\prime}(w)}{1+w^{2}}\right)^{\prime}\right|+2 n \sup _{w \in \mathbb{R}}\left|\left(\frac{w f_{w_{0}}(w)}{\left(1+w^{2}\right)^{2}}\right)^{\prime}\right|\right) E \\
& \times \int_{W \wedge \widetilde{W}}^{W \vee \widetilde{W}}|\widetilde{W}-W|\left|w-\frac{W+\widetilde{W}}{2}\right| d w \\
& \leq \sup _{w \in \mathbb{R}}\left|f_{w_{0}}^{\prime}(w)\right| \sqrt{E\left[1-n E^{W} \frac{(\widetilde{W}-W)^{2}}{1+W^{2}}\right]^{2}}+2 n\left|\frac{E(\widetilde{W}-W)^{2} W f_{w_{0}}(W)}{\left(1+W^{2}\right)^{2}}\right| \\
& +\left(\frac{n}{2} \sup \left|\left(\frac{f_{w_{0}}^{\prime}(w)}{1+w^{2}}\right)^{\prime}\right|+n \sup \left|\left(\frac{w f_{w_{0}}(w)}{\left(1+w^{2}\right)^{2}}\right)^{\prime}\right|\right) E|\widetilde{W}-W|^{3} \\
& \leq 3 \sqrt{E\left[1-n E^{W} \frac{(\widetilde{W}-W)^{2}}{1+W^{2}}\right]^{2}}+2 n\left|\frac{E(\widetilde{W}-W)^{2} W f_{w_{0}}(W)}{\left(1+W^{2}\right)^{2}}\right| \\
& +6 n(\pi+1) E|\widetilde{W}-W|^{3} \\
& \leq 3 \sqrt{E\left[1-n E^{W} \frac{(\widetilde{W}-W)^{2}}{1+W^{2}}\right]^{2}}+2 n\left|\frac{E(\widetilde{W}-W)^{2} W f_{w_{0}}(W)}{\left(1+W^{2}\right)^{2}}\right| \\
& +C \sum_{i=1}^{n} E\left|X_{i}\right|^{3}
\end{aligned}
$$

where the fourth inequality comes from (4) and (5) of Lemma 3.1 and the last inequality comes from (3.25). Since $X_{i}$ and $\tilde{X}_{i}$ are independent and have the same distribution,

$$
E^{W}(\widetilde{W}-W)^{2}=E^{W}\left(\tilde{X}_{I}-X_{I}\right)^{2}=\frac{1}{n} \sum_{i=1}^{n}\left(\tilde{X}_{i}-X_{i}\right)^{2}=\frac{1}{n}\left(\sum_{i=1}^{n} \sigma_{i}^{2}+\sum_{i=1}^{n} X_{i}^{2}\right) .
$$


Hence,

$$
\begin{aligned}
E\left[1-n E^{W} \frac{(\widetilde{W}-W)^{2}}{1+W^{2}}\right]^{2} & =E\left[1-n E^{W} \frac{(\widetilde{W}-W)^{2}}{1+W^{2}}\right]^{2} \\
& =E\left[1-\sum_{i=1}^{n} \frac{\sigma_{i}^{2}+X_{i}^{2}}{1+W^{2}}\right]^{2}
\end{aligned}
$$

Next, we will give a bound of $2 n E(\widetilde{W}-W)^{2}\left(W f_{w_{0}}(W) /\left(1+W^{2}\right)^{2}\right)$.

From Lemma 3.1(1),

$$
\begin{aligned}
\left|2 n E(\widetilde{W}-W)^{2} \frac{W f_{w_{0}}(W)}{\left(1+W^{2}\right)^{2}}\right| & \leq 2 \pi F\left(w_{0}\right)\left(1-F\left(w_{0}\right)\right) \sum_{i=1}^{n} E\left|\tilde{X}_{i}-X_{i}\right|^{2} \\
& =4 \pi F\left(w_{0}\right)\left(1-F\left(w_{0}\right)\right) \sum_{i=1}^{n} \sigma_{i}^{2} \\
\left|2 n E(\widetilde{W}-W)^{2} \frac{W f_{w_{0}}(W)}{\left(1+W^{2}\right)^{2}}\right| & \leq 2 n \pi F\left(w_{0}\right)\left(1-F\left(w_{0}\right)\right) E|\widetilde{W}-W|^{2}|W| \\
& \leq 2 n \pi F\left(w_{0}\right)\left(1-F\left(w_{0}\right)\right) \sqrt{E\left|\tilde{X}_{I}-X_{I}\right|^{4}} \sqrt{E W^{2}} \\
& =8 \pi F\left(w_{0}\right)\left(1-F\left(w_{0}\right)\right) \sqrt{n \sum_{i=1}^{n} \sigma_{i}^{2} \sum_{i=1}^{n} E\left|X_{i}\right|^{4}} .
\end{aligned}
$$

Hence,

$$
\begin{aligned}
& \left|2 n E(\widetilde{W}-W)^{2} \frac{W f_{w_{0}}(W)}{\left(1+W^{2}\right)^{2}}\right| \\
& \quad \leq 4 \pi \min \left\{\sum_{i=1}^{n} \sigma_{i}^{2}, 2 \sqrt{n \sum_{i=1}^{n} \sigma_{i}^{2} \sum_{i=1}^{n} E\left|X_{i}\right|^{4}}\right\} F\left(w_{0}\right)\left(1-F\left(w_{0}\right)\right) .
\end{aligned}
$$

This completes the proof.

4. Proof of Corollary 2.2. Using Taylor's formula, we see that

$$
\begin{array}{cc}
\frac{1}{1+W^{2}}=1-W^{2}+C W^{3} & \text { for some }|C|<1 \\
\frac{1}{\left(1+W^{2}\right)^{2}}=1-2 W^{2}+C W^{3} & \text { for some }|C|<1 .
\end{array}
$$


Hence,

$$
\begin{aligned}
E\left(\frac{1}{1+W^{2}}\right) & \leq \frac{1}{2}+\frac{C}{\sqrt{n}}, \quad E\left(\frac{1}{\left(1+W^{2}\right)^{2}}\right) \leq \frac{C}{\sqrt{n}}, \\
E\left(\frac{\sum_{i=1}^{n} X_{i}^{2}}{1+W^{2}}\right) & =E\left(\sum_{i=1}^{n} X_{i}^{2}\right)-E\left(\sum_{i=1}^{n} X_{i}^{2}\right) W^{2}+C_{1} E\left(\sum_{i=1}^{n} X_{i}^{2}\right) W^{3} \\
& \leq \frac{1}{4}+\frac{C}{\sqrt{n}}, \\
E \frac{\sum_{i=1}^{n} X_{i}^{2}}{\left(1+W^{2}\right)^{2}} & \leq \frac{C}{\sqrt{n}}, \quad E\left(\frac{\sum_{i=1}^{n} X_{i}^{2}}{1+W^{2}}\right)^{2} \leq \frac{C}{n},
\end{aligned}
$$

which implies that

$$
\begin{aligned}
E[1 & \left.-\frac{1}{1+W^{2}}\left(\frac{1}{2}+\sum_{i=1}^{n} X_{i}^{2}\right)\right]^{2} \\
= & 1-E\left(\frac{1}{1+W^{2}}\right)-2 E\left(\frac{\sum_{i=1}^{n} X_{i}^{2}}{1+W^{2}}\right)+\frac{1}{4} E\left(\frac{1}{\left(1+W^{2}\right)^{2}}\right) \\
& +E\left(\frac{\sum_{i=1}^{n} X_{i}^{2}}{\left(1+W^{2}\right)^{2}}\right)+E\left(\frac{\sum_{i=1}^{n} X_{i}^{2}}{1+W^{2}}\right)^{2} \\
\leq & \frac{C}{\sqrt{n}} .
\end{aligned}
$$

Clearly, that

$$
\begin{gathered}
C \sum_{i=1}^{n} E\left|X_{i}\right|^{3} \leq \frac{C}{\sqrt{n}}, \\
4 \pi \min \left\{\sum_{i=1}^{n} \sigma_{i}^{2}, 2 \sqrt{n \sum_{i=1}^{n} \sigma_{i}^{2} \sum_{i=1}^{n} E X_{i}^{4}}\right\} F\left(w_{0}\right)\left(1-F\left(w_{0}\right)\right) \\
\leq C \min \left\{\frac{1}{2}, \sqrt{2} \sqrt{E Y_{i}^{4}}\right\} F\left(w_{0}\right)\left(1-F\left(w_{0}\right)\right) .
\end{gathered}
$$

Hence, by (4.3) and (4.4), the example is proved.

\section{REFERENCES}

[1] R. Arratia, L. Goldstein, and L. Gordon, Two moments suffice for Poisson approximations: the Chen-Stein method, Ann. Probab. 17 (1989), no. 1, 9-25.

[2] , Poisson approximation and the Chen-Stein method, Statist. Sci. 5 (1990), no. 4, 403-434.

[3] P. Baldi and Y. Rinott, On normal approximations of distributions in terms of dependency graphs, Ann. Probab. 17 (1989), no. 4, 1646-1650.

[4] A. D. Barbour, Stein's method and Poisson process convergence, J. Appl. Probab. 25A (1988), 175-184.

[5] _ Stein's method for diffusion approximations, Probab. Theory Related Fields 84 (1990), no. 3, 297-322. 
[6] A. D. Barbour, L. H. Y. Chen, and W.-L. Loh, Compound Poisson approximation for nonnegative random variables via Stein's method, Ann. Probab. 20 (1992), no. $4,1843-1866$.

[7] E. Bolthausen and F. Götze, The rate of convergence for multivariate sampling statistics, Ann. Statist. 21 (1993), no. 4, 1692-1710.

[8] V. Boonyasombut and J. M. Shapiro, The accuracy of infinitely divisible approximations to sums of independent variables with application to stable laws, Ann. Math. Statist. 41 (1970), 237-250.

[9] L. H. Y. Chen, Poisson approximation for dependent trials, Ann. Probab. 3 (1975), no. 3, 534-545.

[10] Stein's method: some perspectives with applications, Probability Towards 2000 (New York, 1995) (L. Accardi and C. C. Heyde, eds.), Lecture Notes in Statist., vol. 128, Springer, New York, 1998, pp. 97-122.

[11] _ Non-uniform bounds in probability approximations using Stein's method, Probability and Statistical Model with Applications: A Volume in Honor of Thephilos Cacoullous (Ch. A. Charalambides, M. V. Koutras, and N. Balakrisshnan, eds.), Chapman\&Hall/CRC Press, Florida, 2000, pp. 3-14.

[12] L. Goldstein and G. Reinert, Stein's method and the zero bias transformation with application to simple random sampling, Ann. Appl. Probab. 7 (1997), no. 4, 935-952.

[13] L. Goldstein and Y. Rinott, Multivariate normal approximations by Stein's method and size bias couplings, J. Appl. Probab. 33 (1996), no. 1, 1-17.

[14] F. Götze, On the rate of convergence in the multivariate CLT, Ann. Probab. 19 (1991), no. 2, 724-739.

[15] T. A. Green, Asymptotic enumeration of generalized Latin rectangles, J. Combin. Theory Ser. A 51 (1989), no. 2, 149-160.

[16] L. Holst and S. Janson, Poisson approximation using the Stein-Chen method and coupling: number of exceedances of Gaussian random variables, Ann. Probab. 18 (1990), no. 2, 713-723.

[17] K. Neammanee, On the rate of convergence of distribution functions of sums of reciprocals of logarithm of random variables to the Cauchy distribution, to appear.

[18] J. M. Shapiro, On the rate of convergence of distribution functions of sums of reciprocals of random variables to the Cauchy distribution, Houston J. Math. 4 (1978), no. 3, 439-445.

[19] C. Stein, A bound for the error in the normal approximation to the distribution of a sum of dependent random variables, Proceedings of the Sixth Berkeley Symposium on Mathematical Statistics and Probability (Univ. California, Berkeley, Calif., 1970/1971), Vol. II: Probability theory (California), Univ. California Press, 1972, pp. 583-602.

[20] - Approximate Computation of Expectations, Institute of Mathematical Statistics Lecture Notes-Monograph Series, vol. 7, Institute of Mathematical Statistics, California, 1986.

K. Neammanee: Department of Mathematics, Faculty of Science, Chulalongkorn University, Bangkok 10330, Thailand

E-mail address: kritsana.n@chu1a.ac.th 


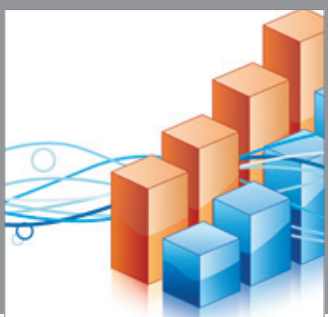

Advances in

Operations Research

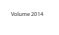

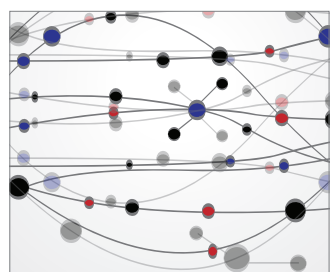

\section{The Scientific} World Journal
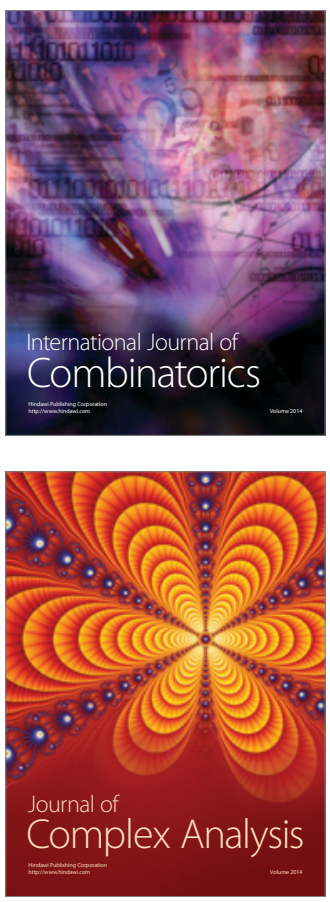

International Journal of

Mathematics and

Mathematical

Sciences
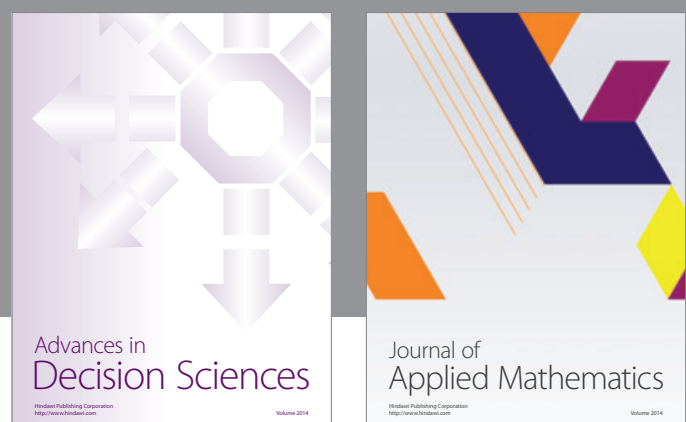

Journal of

Applied Mathematics
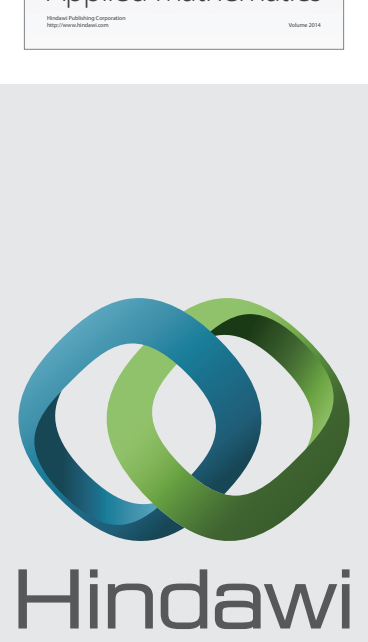

Submit your manuscripts at http://www.hindawi.com
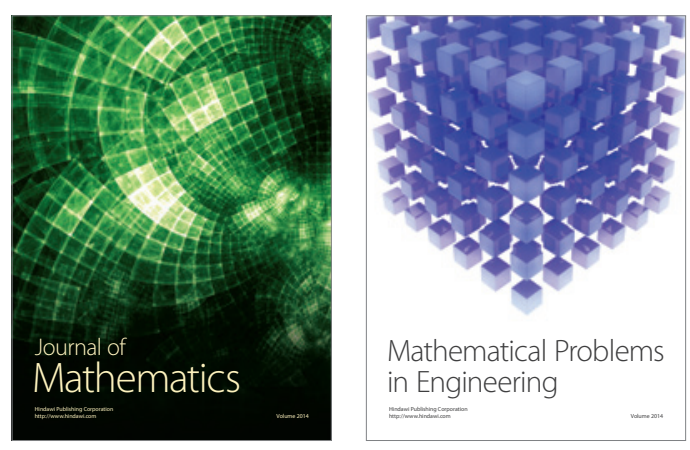

Mathematical Problems in Engineering
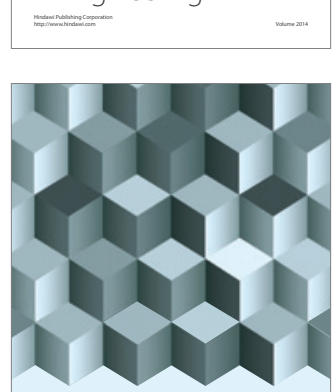

Journal of

Function Spaces
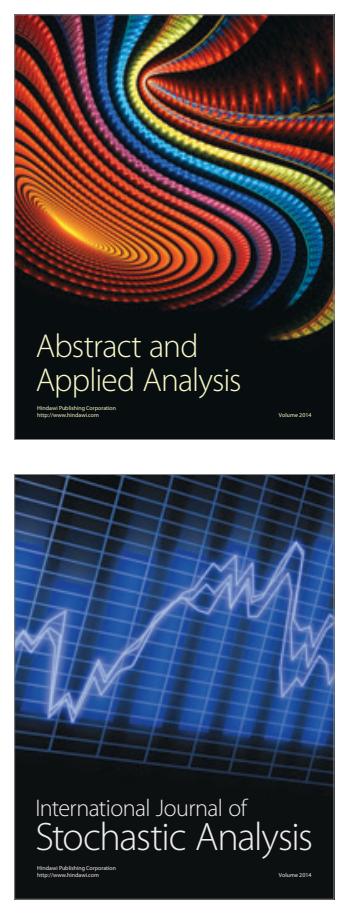

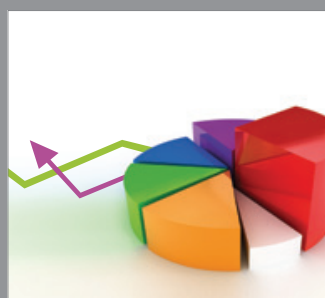

ournal of

Probability and Statistics

Promensencen
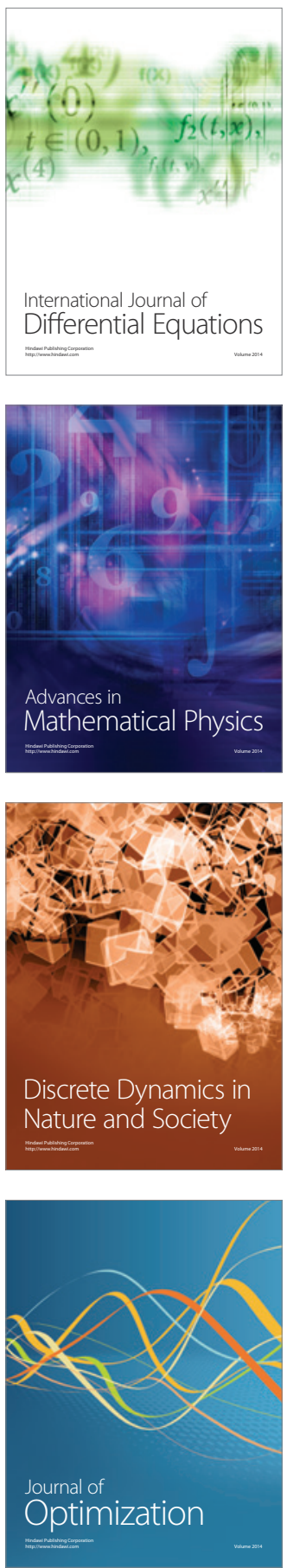